\title{
Numerical and analytical study of drug release from a biodegradable viscoelastic platform
}

\author{
E. Azhdari, J. A. Ferreira, Paula de Oliveira, P.M. da Silva \\ CMUC-Department of Mathematics, University of Coimbra.
}

November 30, 2017

\begin{abstract}
A mathematical model to simulate drug delivery from a viscoelastic erodible matrix is presented in this paper. The drug is initially distributed in the matrix which is in contact with water. The entrance of water in the material changes the molecular weight and bulk erosion can be developed depending on how fast is this entrance and how fast degradation occurs. The viscoelastic properties of the matrix also change in the presence of water as the molecular weight changes. The model is represented by a system of quasi linear partial differential equations that take into account different phenomena: the uptake of water, the decreasing of the molecular weight, the viscolestic behaviour, the dissolution of the solid drug and the delivery of the dissolved drug. Numerical simulations illustrating the behaviour of the model are included.
\end{abstract}

Keywords:Drug delivery; molecular weight; bulk erosion; viscoelastic behaviour; numerical simulation.

\section{Mathematical model}

We consider a biodegradable viscoelastic polymeric matrix, $\Omega \subseteq \mathbb{R}^{2}$, with boundary $\partial \Omega$ and containing a limited amount of drug. The matrix enters in contact with water and as the water diffuses into the matrix, a hydratation process, that modifies the viscoelastic properties of the polymer, takes place. The molecular weight decreases and the drug starts to dissolve.

In [15] a system that describes the sorption of water, by a loaded erodible matrix and the release of drug was proposed. However the viscoelastic properties of the matrix were not considered. In this paper we present a general model, which generalizes the model in [15], by considering the viscoelastic behaviour of the polymer (see for instance [1],[2], [7],[11], [16], [18]).

We consider a system of partial differential equations (PDE's) that describe the whole process: the entrance of water into the polymer and its consumption in the hydrolysis process; the decreasing of the molecular weight; the evolution of the stress and strain; the dissolution and the diffusion of the dissolved drug. The system reads

$$
\begin{cases}\frac{\partial C_{W}}{\partial t}=\nabla \cdot\left(D_{W} \nabla C_{W}\right)+\nabla \cdot\left(D_{v} \nabla \sigma\right)-k C_{W} M & \text { in } \Omega \times(0, T], \\ \frac{\partial M}{\partial t}=-\widetilde{k} C_{W} M & \text { in } \Omega \times(0, T], \\ \frac{\partial \sigma}{\partial t}+\frac{E(M)}{\mu(M)} \sigma=-E(M) \frac{\partial C_{W}}{\partial t} & \text { in } \Omega \times(0, T], \\ \frac{\partial C_{S}}{\partial t}=-k_{d i s} C_{S n} C_{A n} C_{W n} & \text { in } \Omega \times(0, T], \\ \frac{\partial C_{A}}{\partial t}=\nabla \cdot\left(D(M) \nabla C_{A}\right)+k_{d i s} C_{S n} C_{A n} C_{W n} & \text { in } \Omega \times(0, T] .\end{cases}
$$

\footnotetext{
${ }^{1}$ E. Azhdari, email:ebrahim@mat.uc.pt, CMUC-Department of Mathematics, University of Coimbra.

${ }^{1}$ J. A. Ferreira, email:ferreira@mat.uc.pt, CMUC-Department of Mathematics, University of Coimbra.

${ }^{1}$ Paula de Oliveira, email:poliveir@mat.uc.pt, CMUC-Department of Mathematics, University of Coimbra.

${ }^{2}$ P.M. da Silva, email: pascals@isec.pt, Instituto Politécnico de Coimbra, ISEC, DFM, Rua Pedro Nunes, 3030-199 Coimbra, Portugal. CMUC.
} 
In (1) $C_{W}, C_{S}$ and $C_{A}$ represent the concentration of water, solid drug and dissolved drug in the polymeric matrix, respectively, $M$ is the molecular weight of the polymer and $\sigma$ is the stress response to the strain exerted by the water molecules.

The first diffusion-reaction equation of (1) describes the diffusion of water into the matrix and its consumption in the hydrolysis. In this equation $D_{W}$ represents the diffusion tensor of water in the polymeric matrix. We consider an isotropic medium where the diffusion tensors are diagonal with equal diagonal elements. For example, $D_{W}=D_{W} I$, where $I$ is the $2 \times 2$ identity matrix. The viscoelastic opposition to the water entrance is represented by $\nabla \cdot\left(D_{v} \nabla \sigma\right)$ where $D_{v}$ is a viscoelastic diffusion tensor. This term states that the polymer acts as a barrier to the diffusion of water into the polymeric matrix. The term $-k C_{W} M$ represents the consumption of water in the hydrolysis of the polymer. The molecular weight loss is represented by $-\widetilde{k} C_{W} M([3],[5],[6],[8])$.

Since the water diffuses into the polymeric matrix the molecules of water react with the polymer and the bounds between the polymeric chains are broken leading to a decrease in the molecular weight of the matrix. This process is described by the second equation of (1) ([15]).

We assume that the viscoelastic behaviour of the polymer can be modelled by Maxwell fluid model

$$
\frac{\partial \sigma}{\partial t}+\frac{E}{\mu} \sigma=E \frac{\partial \epsilon}{\partial t},
$$

where $E$ represents the Young modulus of the material, $\mu$ is its viscosity and $\epsilon$ is the strain produced by the water molecules. We assume that the strain and the concentration of water are proportional, that is, there exists $k_{1}>0$ such that $\epsilon=k_{1} C_{W}$, where $k_{1}$ stands for a dimensional constant. This relation is a linear approximation of $\epsilon=f(C)$ established in [8]. As the polymer acts as a barrier to the entrance of the water, then $\sigma$ and $\epsilon$ are of opposite sign, and a minus sign should be considered in the right hand side of $(2)$.

Based on the results presented for instance in [1], [2], [7], [11], [16] and [18], we assume that the Young modulus and the viscosity depend on the molecular weight. In fact the Young modulus varies significantly in a biodegradable polymeric matrix due to the heterogeneous nature of the hydrolysis reaction that leads to the cleavages of the polymeric chains. As the degradation processes evolves, the Young modulus decreases ([13]). Moreover a functional relation between the viscosity and the molecular weight represented by Mark-Houwink equation ([14]) is applied. The expressions used to represent the behaviours of $E(M)$ and $\mu(M)$ are $E(M)=E_{0} M^{\alpha}$ and $\mu(M)=\mu_{0} M^{\beta}$ where $E_{0}, \mu_{0}, \alpha$ and $\beta$ are constant $([14],[13],[3])$.

The evolution in time of the solid drug is described by the fourth equation of (1) where $k_{d i s}$ is the dissolution rate, $C_{S n}$ is the normalized concentration of solid drug in the polymeric matrix, $C_{A n}$ is the difference between the dissolved drug concentration and its maximum solubility $\left(C_{A m x}\right)$, normalized by $C_{A m x}, C_{W n}$ is the normalized concentration of water $\left(\frac{C_{W}}{C_{W o u t}}\right)$. In this last expression $C_{W o u t}$ is the concentration of water outside of the polymeric matrix. The evolution of the concentration of dissolved drug in the matrix is defined by the last equation of (1) where Fick's law and the dissolution source were taken into account.

As the degradation occurs the molecular weight decreases and the permeability of the polymer increases. This leads to an increase of the diffusion coefficient ([17]) that can be represented by

$$
D(M)=D_{A} e^{\bar{k} \frac{M_{0}-M}{M_{0}}},
$$

where $D_{A}$ is the diffusion coefficient of the drug in the non hydrolyzed polymer, $M_{0}$ is its initial molecular weight and $\bar{k}$ is a positive constant.

System (1) is completed with the initial conditions

$$
\begin{cases}C_{W}(0)=0 & \text { in } \Omega, \\ \sigma(0)=\sigma_{0} & \text { in } \Omega, \\ M(0)=M_{0} & \text { in } \Omega, \\ C_{S}(0)=C_{S 0} & \text { in } \Omega, \\ C_{A}(0)=0 & \text { in } \Omega,\end{cases}
$$


where $\sigma_{0}$ represents the initial stress of the polymer and $C_{S 0}$ is the initial concentration of solid drug in the polymeric matrix.

Degradation of the polymeric matrix can be one of the two types: surface and bulk. Surface degradation occurs because degradation is faster than the entrance of water in the system. In this case the cleavage of polymeric chains occurs mainly in the outermost polymeric layers. Bulk degradation occurs when the degradation is slower than the water uptake. The entire system is rapidly hydrated and polymeric chains are cleaved through all the polymeric structure ([17]).

In what follows we assume that bulk degradation occurs and that the physical domain is maintained during all diffusion process. The entrance of water occurs due to the difference of concentrations in the polymer and in the medium. Then the system (1) and the initial conditions are coupled with the following boundary condition

$$
\begin{cases}J \cdot \eta=A_{c}\left(C_{W}-C_{W o u t}\right) & \text { on } \partial \Omega \times(0, T], \\ C_{A}=0 & \text { on } \partial \Omega \times(0, T],\end{cases}
$$

where $J$ represents the flux defined by $J=-D_{W} \nabla C_{W}-D_{v} \nabla \sigma, \eta$ is the unit outward normal to $\partial \Omega, A_{c}$ is the permeability constant and $C_{W o u t}$ denotes the water concentration out of the polymeric matrix.

The aim of this paper is to present a numerical method to solve (1), (3) and (4) and to study the qualitative behaviour of the numerical solution. In Section 2 a stability analysis of the mathematical model is studied. Implicit-Explicit method (IMEX) is introduced and its convergence is numerically studied in Secton 3. In Section 4 the qualitative behavior of the solution is analysed. Finally in Section 5 we present some conclusions.

\section{Stability analysis}

In order to simplify the presentation, we assume in this section that $E$ and $\mu$ are constant. We also assume that the diffusion tensor is only space dependent.

To gain some insight on the stability behaviour of the initial value problem (1),(3) and (4) we study in what follows the stability of a linearization of (1) for short and long times. For short times we linearize the system in the neighborhood of the initial state; for large times the system is linearized in the neighborhood of the steady state solution. Let $\tilde{C}_{W}, \tilde{M}, \tilde{C}_{A}$ and $\tilde{C}_{S}$ be a solution of (1). The linearized system at this solution can be written in the following form

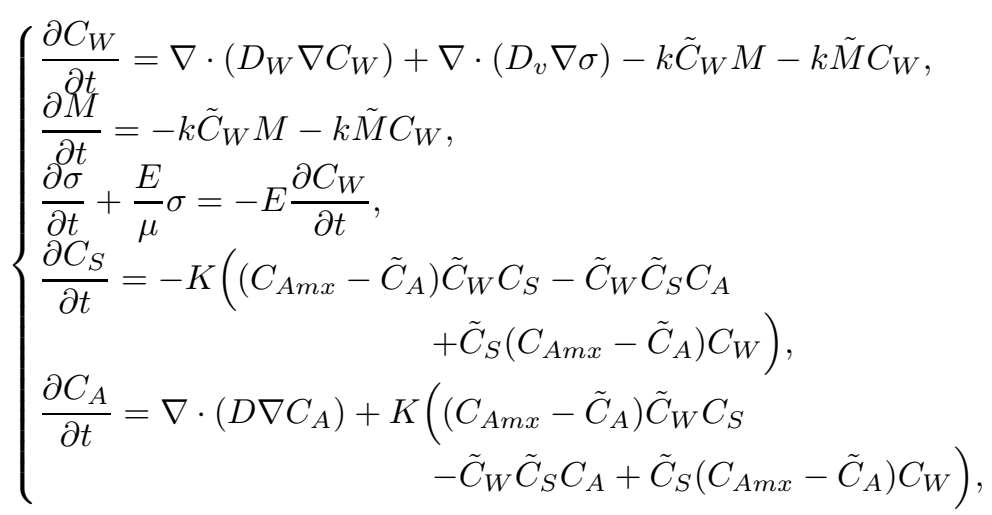

where $K=\frac{k_{d i s}}{C_{S_{0}} C_{A m x} C_{W o u t}}$ is a constant.

For small times the concentration of water and dissolved drug is very small so we consider

$$
\tilde{C}_{W}=0, \tilde{C}_{A}=0, \tilde{C}_{S}=C_{S 0}, \tilde{M}=M_{0} .
$$

For large times, that is when the matrix is practically degraded and the drug released, we assume

$$
\tilde{M}=0, \tilde{C}_{W}=C_{W o u t}, \tilde{C}_{S}=0, \tilde{C}_{A}=0 .
$$

Solution (6) defines the state of the system as $t \rightarrow 0$. So for small times, the stability of system (1) is obtained studying the stability of (5) and (4) when (6) is considered. When $t \rightarrow+\infty$, the solution of 
system (1) approaches the steady solution (7). In fact, phenomenologically, the molecular weight decreases and vanishes, the concentration of water goes to the equilibrium, that is $C_{W o u t}$, the concentrations of solid and dissolved drug inside of the polymeric matrix vanish.

Stability for short times: To study the stability of (4) and (5), we consider the difference of two solutions, which we represent by the same notations $C_{W}, \sigma, M, C_{S}, C_{A}$. In this case we have

$$
\begin{cases}\frac{\partial C_{W}}{\partial t}=\nabla \cdot\left(D_{W} \nabla C_{W}\right)+\nabla \cdot\left(D_{v} \nabla \sigma\right) & \\ \frac{\partial M}{\partial t}=-k M_{0} C_{W} & \text { in } \Omega \times(0, T], \\ \frac{\partial \sigma}{\partial t}+\frac{E}{\mu} \sigma=-E \frac{\partial C_{W}}{\partial t} & \text { in } \Omega \times(0, T], \\ \frac{\partial C_{S}}{\partial t}=-\frac{k_{d i s}}{C_{W o u t}} C_{W} & \text { in } \Omega \times(0, T], \\ \frac{\partial C_{A}}{\partial t}=\nabla \cdot\left(D \nabla C_{A}\right)+\frac{k_{\text {dis }}}{C_{W o u t}} C_{W} & \text { in } \Omega \times(0, T],\end{cases}
$$

where $T>0$ is fixed, with the boundary conditions

$$
\begin{cases}J \cdot \eta=A_{c} C_{W} & \text { on } \partial \Omega \times(0, T], \\ C_{A}=0 & \text { on } \partial \Omega \times(0, T] .\end{cases}
$$

In what follows we use the energy method to analyze (8) and (9) complemented with the initial condition

$$
\begin{cases}C_{W}(0)=C_{W 0} & \text { in } \Omega, \\ \sigma(0)=\sigma_{0} & \text { in } \Omega, \\ M(0)=M_{0} & \text { in } \Omega, \\ C_{S}(0)=C_{S 0} & \text { in } \Omega, \\ C_{A}(0)=C_{A 0} & \text { in } \Omega .\end{cases}
$$

From the third equation of (8) we easily get

$$
\begin{aligned}
\sigma=\frac{E^{2}}{\mu} \int_{0}^{t} e^{-\frac{E}{\mu}(t-s)} C_{W}(s) d s & -E C_{W}+E C_{W}(0) e^{-\frac{E}{\mu} t} \\
& +\sigma(0) e^{-\frac{E}{\mu} t}, t \geq 0
\end{aligned}
$$

and using this equality in the first equation of (8) we obtain for $C_{W}$ the following equation

$$
\begin{aligned}
& \frac{\partial C_{W}}{\partial t}=\nabla \cdot\left(D_{1} \nabla C_{W}\right)+\int_{0}^{t} e^{-\frac{E}{\mu}(t-s)} \nabla \cdot\left(D_{2} \nabla C_{W}(s)\right) d s-k M_{0} C_{W} \\
& +E e^{-\frac{E}{\mu} t} \nabla \cdot\left(D_{v} \nabla C_{W}(0)\right)+e^{-\frac{E}{\mu} t} \nabla \cdot\left(D_{v} \nabla \sigma(0)\right),
\end{aligned}
$$

where

$$
D_{1}=D_{W}-E D_{v}, \quad D_{2}=\frac{E^{2}}{\mu} D_{v}
$$

We assume that, in (13), $D_{W}, D_{v}$ and $D$ are $2 \times 2$ diagonal matrices and $E$ and $\mu$ are such that the entries of $D_{1}$ and $D_{2}$ are positive and satisfy the following conditions:

$$
D_{1, j j} \geq D_{\min }, \quad D_{2, j j}, D_{v, j j} \leq D_{\max }, \quad D_{j j} \geq D_{0}, \quad \text { for } j=1,2 .
$$

Let $V=H^{1}(\Omega) \times\left(L^{2}(\Omega)\right)^{2} \times H_{0}^{1}(\Omega)$ and let $\left(C_{W}, M, C_{S}, C_{A}\right) \in V$ be such that $\frac{\partial C_{W}}{\partial t}, \frac{\partial M}{\partial t}, \frac{\partial C_{S}}{\partial t}$, $\frac{\partial C_{A}}{\partial t} \in L^{2}(\Omega)$ and that (10) holds. Then multiplying scalarly the equations in (8) by test functions, where the first and the third equations are replaced by (12), we have 


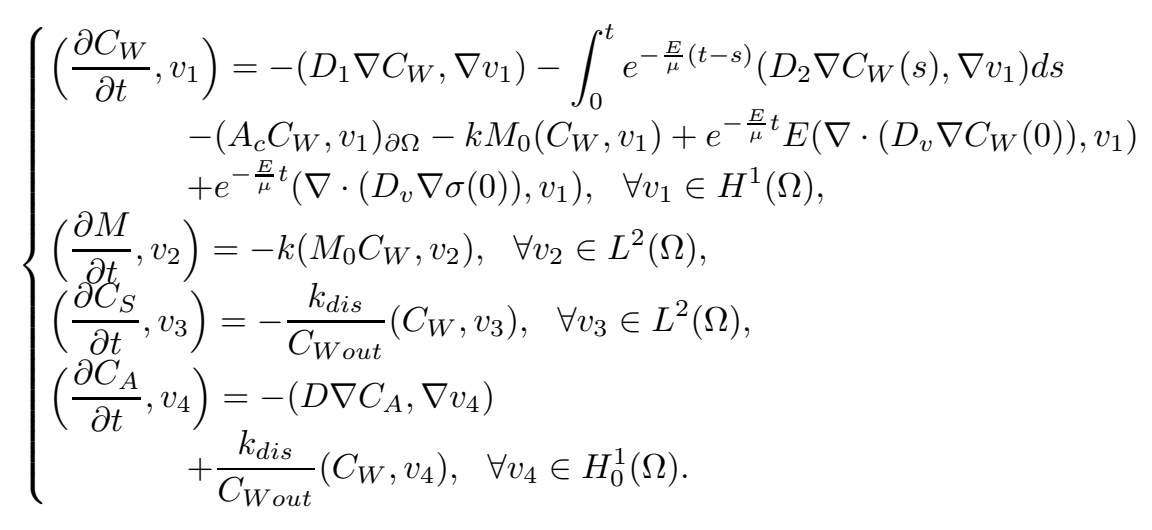

In (15) the same notation is used to represent the usual inner products in $L^{2}(\Omega)$ and $\left(L^{2}(\Omega)\right)^{2}$. We establish in what follows an estimate for the energy functional

$$
\mathcal{E}(t)=E_{C M}(t)+\int_{0}^{t}\left(\left\|\nabla C_{W}(s)\right\|^{2}+\left\|\nabla C_{A}(s)\right\|^{2}\right) d s, \quad t \in[0, T]
$$

with

$$
E_{C M}(t)=\left\|C_{W}(t)\right\|^{2}+\|M(t)\|^{2}+\left\|C_{S}(t)\right\|^{2}+\left\|C_{A}(t)\right\|^{2}
$$

where the same notation $\|$.$\| was used to represent the norm induced by the usual inner products in$ $L^{2}(\Omega)$ and $\left(L^{2}(\Omega)\right)^{2}$.

Theorem 1 Let $\left(C_{W}, M, C_{S}, C_{A}\right) \in V$ be a solution of the variational problem (15). Then

$$
\begin{array}{r}
\mathcal{E}(t) \leq \frac{1}{\min \left\{1,2\left(D_{\min }-\epsilon_{1}^{2}\right), 2 D_{0}\right\}}\left(\frac { \mu } { 4 E \epsilon _ { 2 } ^ { 2 } } \left(E^{2}\left\|\nabla \cdot\left(D_{v} \nabla C_{W}(0)\right)\right\|^{2}\right.\right. \\
\left.\left.+\left\|\nabla \cdot\left(D_{v} \nabla \sigma(0)\right)\right\|^{2}\right)+E_{C M}(0)\right) e^{\bar{c} t}
\end{array}
$$

where $\epsilon_{1} \neq 0$ satisfies

$$
D_{\min }-\epsilon_{1}^{2}>0
$$

and

$$
\bar{c}=\frac{\max \left\{\frac{D_{\text {max }}^{2} \mu}{4 \epsilon_{1}^{2} E}, 2 \epsilon_{3}^{2}\left(k^{2} M_{0}^{2}+2 \frac{k_{\text {dis }}^{2}}{C_{\text {Wout }}^{2}}\right)+4 \epsilon_{2}^{2}-2 k M_{0}, \frac{1}{2 \epsilon_{3}^{2}}\right\}}{\min \left\{1,2\left(D_{\text {min }}-\epsilon_{1}^{2}\right), 2 D_{0}\right\}}
$$

being $\epsilon_{2}, \epsilon_{3} \neq 0$ arbitrary constants.

Proof: Taking in (15) $v_{1}=C_{W}, v_{2}=M, v_{3}=C_{S}$ and $v_{4}=C_{A}$, we easily obtain from the first equation

$$
\begin{array}{r}
\frac{1}{2} \frac{d}{d t}\left\|C_{W}\right\|^{2}=-\left(D_{1} \nabla C_{W}, \nabla C_{W}\right) \\
-\int_{0}^{t} e^{-\frac{E}{\mu}(t-s)}\left(D_{2} \nabla C_{W}(s), \nabla C_{W}\right) d s \\
-A_{c}\left\|C_{W}\right\|_{\partial \Omega}^{2}+e^{-\frac{E}{\mu} t} E\left(\nabla \cdot\left(D_{v} \nabla C_{W}(0)\right), C_{W}\right) \\
+e^{-\frac{E}{\mu} t}\left(\nabla \cdot\left(D_{v} \nabla \sigma(0)\right), C_{W}\right)-k M_{0}\left\|C_{W}\right\|^{2},
\end{array}
$$


where $\|.\|_{\partial \Omega}$ denotes the usual norm in $L^{2}(\partial \Omega)$. The remaining three equations of (15) lead to

$$
\begin{gathered}
\frac{1}{2} \frac{d}{d t}\|M\|^{2}=-k M_{0}\left(C_{W}, M\right), \\
\frac{1}{2} \frac{d}{d t}\left\|C_{S}\right\|^{2}=-\frac{k_{d i s}}{C_{W \text { out }}}\left(C_{W}, C_{S}\right),
\end{gathered}
$$

and

$$
\frac{1}{2} \frac{d}{d t}\left\|C_{A}\right\|^{2}=-\left(D \nabla C_{A}, \nabla C_{A}\right)+\frac{k_{d i s}}{C_{W o u t}}\left(C_{W}, C_{A}\right) .
$$

For any non zero constants $\epsilon_{1}, \epsilon_{2}$ and $\epsilon_{3}$ we have the following inequalities

$$
\begin{gathered}
-\int_{0}^{t} e^{-\frac{E}{\mu}(t-s)}\left(D_{2} \nabla C_{W}(s), \nabla C_{W}\right) d s \leq \\
\quad \epsilon_{1}^{2}\left\|\nabla C_{W}\right\|^{2} \\
+\frac{D_{\text {max }}^{2} \mu}{8 \epsilon_{1}^{2} E} \int_{0}^{t}\left\|\nabla C_{W}(s)\right\|^{2} d s, \\
\leq \frac{1}{4 \epsilon_{2}^{2}} e^{-2 \frac{E}{\mu} t}\left(E^{2}\left\|\nabla \cdot\left(D_{v} \nabla C_{W}(0)\right)\right\|^{2}+\left\|\nabla \cdot\left(D_{v} \nabla \sigma(0)\right)\right\|^{2}\right)+2 \epsilon_{2}^{2}\left\|C_{w}\right\|^{2}, \\
k M_{0}\left(C_{W}, M\right) \leq k^{2} M_{0}^{2} \epsilon_{3}^{2}\left\|C_{W}\right\|^{2}+\frac{1}{4 \epsilon_{3}^{2}}\|M\|^{2}, \\
\frac{k_{\text {dis }}}{C_{W \text { out }}}\left(C_{W}, C_{S}\right) \leq \frac{k_{\text {dis }}^{2}}{C_{W \text { out }}^{2}} \epsilon_{3}^{2}\left\|C_{W}\right\|^{2}+\frac{1}{4 \epsilon_{3}^{2}}\left\|C_{S}\right\|^{2}, \\
\frac{k_{\text {dis }}}{C_{W \text { out }}}\left(C_{W}, C_{A}\right) \leq \frac{k_{\text {dis }}^{2}}{C_{W \text { out }}^{2}} \epsilon_{3}^{2}\left\|C_{W}\right\|^{2}+\frac{1}{4 \epsilon_{3}^{2}}\left\|C_{A}\right\|^{2} .
\end{gathered}
$$

Summing up the preceeding three equations we obtain

$$
\begin{aligned}
\frac{d}{d t} E_{C M} & +2\left(D_{\min }-\epsilon_{1}^{2}\right)\left\|\nabla C_{W}\right\|^{2}+2 D_{0}\left\|\nabla C_{A}\right\|^{2} \\
& \leq \frac{D_{\max }^{2} \mu}{4 \epsilon_{1}^{2} E} \int_{0}^{t}\left\|\nabla C_{W}(s)\right\|^{2} d s \\
& +\left(2 \epsilon_{3}^{2}\left(k^{2} M_{0}^{2}+2 \frac{k_{d i s}^{2}}{C_{W o u t}^{2}}\right)+4 \epsilon_{2}^{2}-2 k M_{0}\right)\left\|C_{W}\right\|^{2} \\
& +\frac{1}{2 \epsilon_{3}^{2}}\left(\|M\|^{2}+\left\|C_{S}\right\|^{2}+\left\|C_{A}\right\|^{2}\right) \\
& +\frac{1}{2 \epsilon_{2}^{2}} e^{-2 \frac{E}{\mu} t}\left(E^{2}\left\|\nabla \cdot\left(D_{v} \nabla C_{W}(0)\right)\right\|^{2}+\left\|\nabla \cdot\left(D_{v} \nabla \sigma(0)\right)\right\|^{2}\right),
\end{aligned}
$$

where $E_{C M}$ is defined in (17). If we fix $\epsilon_{1}$ satisfying (19) then

$$
\begin{gathered}
\mathcal{E}(t) \leq \bar{c} \int_{0}^{t} \mathcal{E}(s) d s+\frac{1}{\min \left\{1,2\left(D_{\min }-\epsilon_{1}^{2}\right), 2 D_{0}\right\}}\left(\frac{\mu}{4 E \epsilon_{2}^{2}}\right. \\
\left.\left(E^{2}\left\|\nabla \cdot\left(D_{v} \nabla C_{W}(0)\right)\right\|^{2}+\left\|\nabla \cdot\left(D_{v} \nabla \sigma(0)\right)\right\|^{2}\right)+E_{C M}(0)\right),
\end{gathered}
$$


where $\bar{c}$ is defined by (20). Finally by using Gronwall's Lemma we obtain (18).

The energy estimate (18) leads to the uniqueness of solution of the variational problem (15) and (10). It enables also to conclude the stability of such solution in bounded time intervals. These results hold provided that the initial data are smooth enough.

Stability for large times: To analyze the stability of the initial boundary value problem (1),(3) and (4) for large times we consider system (5), that arise from the linearization of system (1) in the neighborhood of the steady solution defined by (7). That is, we study the stability of the initial boundary value problem

$$
\begin{cases}\frac{\partial C_{W}}{\partial t}=\nabla \cdot\left(D_{W} \nabla C_{W}\right)+\nabla \cdot\left(D_{v} \nabla \sigma\right)-k C_{W \text { out }} M & \text { in } \Omega \times(0, T], \\ \frac{\partial M}{\partial t}=-k C_{W \text { out }} M & \text { in } \Omega \times(0, T], \\ \frac{\partial \sigma}{\partial t}+\frac{E}{\mu} \sigma=-E \frac{\partial C_{W}}{\partial t} & \text { in } \Omega \times(0, T], \\ \frac{\partial C_{S}}{\partial t}=-\frac{k_{d i s}}{C_{S 0}} C_{S} & \text { in } \Omega \times(0, T], \\ \frac{\partial C_{A}}{\partial t}=\nabla \cdot\left(D \nabla C_{A}\right)+\frac{k_{d i s}}{C_{S 0}} C_{S} & \text { in } \Omega \times(0, T],\end{cases}
$$

where $T>0$ is fixed, with initial conditions

$$
\begin{cases}C_{W}(0)=C_{W, \infty} & \text { in } \Omega, \\ \sigma(0)=\sigma_{\infty} & \text { in } \Omega, \\ M(0)=M_{\infty} & \text { in } \Omega, \\ C_{S}(0)=C_{S \infty} & \text { in } \Omega, \\ C_{A}(0)=C_{A, \infty} & \text { in } \Omega,\end{cases}
$$

and boundary conditions (9).

From the third equation of (22) we easily get an expression for the stress $\sigma$ analogous to (11). Replacing then that expression in the first equation of (22) we obtain

$$
\left\{\begin{aligned}
\frac{\partial C_{W}}{\partial t} & =\nabla \cdot\left(D_{1} \nabla C_{W}\right)+\int_{0}^{t} e^{-\frac{E}{\mu}(t-s)} \nabla \cdot\left(D_{2} \nabla C_{W}(s)\right) d s & & \\
& -k C_{W \text { out }} M+E e^{-\frac{E^{\mu}}{\mu}} \nabla \cdot\left(D_{v} \nabla C_{W}(0)\right) & & \text { in } \Omega \times(0, T], \\
& +e^{-\frac{E}{\mu} t} \nabla \cdot\left(D_{v} \nabla \sigma(0)\right) & & \text { in } \Omega \times(0, T], \\
\frac{\partial M}{\partial t}= & -k C_{W o u t} M & & \text { in } \Omega \times(0, T], \\
\frac{\partial C_{S}}{\partial t} & =-\frac{k_{d i s}}{C_{S 0}} C_{S} & & \text { in } \Omega \times(0, T], \\
\frac{\partial C_{A}}{\partial t} & =\nabla \cdot\left(D \nabla C_{A}\right)+\frac{k_{d i s}}{C_{S 0}} C_{S} & & \text { r }
\end{aligned}\right.
$$

where $D_{1}$ and $D_{2}$ are given by (13). The original initial boundary value problem (22), (9) and (23) is then replaced by (24), completed with (23) and (9).

In what follows we consider the weak formulation of (24), (23) and (9) defined by the variational problem: 
Find $\left(C_{W}, M, C_{S}, C_{A}\right) \in V$ such that $\frac{\partial C_{W}}{\partial t}, \frac{\partial M}{\partial t}, \frac{\partial C_{S}}{\partial t}, \frac{\partial C_{A}}{\partial t} \in L^{2}(\Omega)$, and (23) holds and

$$
\left\{\begin{aligned}
&\left(\frac{\partial C_{W}}{\partial t}, v_{1}\right)=-(\left.D_{1} \nabla C_{W}, \nabla v_{1}\right)-\int_{0}^{t} e^{-\frac{E}{\mu}(t-s)}\left(D_{2} \nabla C_{W}(s), \nabla v_{1}\right) d s \\
&-\left(A_{c} C_{W}, v_{1}\right) \partial \Omega-k C_{W o u t}\left(M, v_{1}\right) \\
&+E e^{-\frac{E}{\mu} t}\left(\nabla \cdot\left(D_{v} \nabla C_{W}(0)\right), v_{1}\right) \\
&+e^{-\frac{E}{\mu} t}\left(\nabla \cdot\left(D_{v} \nabla \sigma(0)\right), v_{1}\right), \quad \forall v_{1} \in H^{1}(\Omega) \\
&\left(\frac{\partial M}{\partial t}, v_{2}\right)=-k C_{W o u t}\left(M, v_{2}\right), \quad \forall v_{2} \in L^{2}(\Omega), \\
&\left(\frac{\partial C_{S}}{\partial t}, v_{3}\right)=-\frac{k_{d i s}}{C_{S 0}}\left(C_{S}, v_{3}\right), \quad \forall v_{3} \in L^{2}(\Omega) \\
&\left(\frac{\partial C_{A}}{\partial t}, v_{4}\right)=-\left(D \nabla C_{A}, \nabla v_{4}\right) \\
&+\frac{k_{d i s}}{C_{S 0}}\left(C_{S}, v_{4}\right), \quad \forall v_{4} \in H_{0}^{1}(\Omega)
\end{aligned}\right.
$$

Following the proof of Theorem 1 it can be established an upper bound for $\mathcal{E}(t)$ analogous to the one defined by (16).

Theorem 2 If $\left(C_{W}, M, C_{S}, C_{A}\right) \in V$ is a solution of the variational problem (25), then

$$
\begin{aligned}
\mathcal{E}(t) & \leq \frac{1}{\min \left\{1,2\left(D_{\min }-\epsilon_{1}^{2}\right), 2 D_{0}\right\}}\left(E_{C M}(0)\right. \\
& \left.+\frac{\mu}{4 E \epsilon_{2}^{2}}\left(E^{2}\left\|\nabla \cdot\left(D_{v} \nabla C_{W}(0)\right)\right\|^{2}+\left\|\nabla \cdot\left(D_{v} \nabla \sigma(0)\right)\right\|^{2}\right)\right) e^{\bar{c} t}, t \geq 0,
\end{aligned}
$$

where $\epsilon_{1}$ is fixed by (19),

$$
\bar{c}=\frac{\max \left\{\frac{\mu D_{\max }^{2}}{4 \epsilon_{1}^{2} E}, 2 k C_{W o u t}\left(\frac{k C_{W \text { out }}}{4 \epsilon_{2}^{2}}-1\right), 2 \frac{k_{\text {dis }}}{C_{S_{0}}}\left(\frac{k_{\text {dis }}}{C_{S 0}} \epsilon_{3}^{2}-1\right), \frac{1}{2 \epsilon_{3}^{2}}, 6 \epsilon_{2}^{2}\right\}}{\min \left\{1,2\left(D_{\min }-\epsilon_{1}^{2}\right), 2 D_{0}\right\}},
$$

and $\epsilon_{2}, \epsilon_{3}$ are arbitrary nonzero constant.

From Theorem 2 we conclude the uniqueness of the solution of (25) and (23) and its stability for bounded time intervals, provided that the initial data are smooth enough.

As the model is nonlinear we carried out a stability analysis based on a local linearization in the neighborhood of the steady state solutions for short and large times. The stability in both cases was established under the positiveness of the diagonal entries of $D_{1}$ defined in (13). This is a central issue meaning that the Fickian diffusion must dominate the non Fickian one. Due to the interpretation of viscoelasticity that we propose - as representing a barrier to the penetration of water - such assumption is physically sound.

\section{Numerical method}

In this section we introduce a Implicit-Explicit finite difference method to solve (1), (3), (4). Let $\Omega$ be the square $(0, L) \times(0, L)$, where $L$ represents the thickness of the polymer. We fix $h>0$ and we define in $\bar{\Omega}$ the grid

$$
\begin{array}{r}
\bar{\Omega}_{h}=\left\{\left(x_{i}, y_{j}\right), i, j=0, \ldots, N, x_{0}, y_{0}=0, x_{N}, y_{N}=L,\right. \\
\left.x_{i}-x_{i-1}=h, y_{j}-y_{j-1}=h, i, j=1, \ldots, N\right\} .
\end{array}
$$

By $\Omega_{h}$ and $\partial \Omega_{h}$ we represent the mesh nodes of $\bar{\Omega}_{h}$ that are in $\Omega$ and on the boundary $\partial \Omega$, respectively. Let $u_{h}$ and $v_{h}$ be grid functions defined in $\bar{\Omega}_{h}$. To discretize the spatial derivatives we introduce the 
second order finite difference operator

$$
\begin{aligned}
& \quad D_{x}^{*}\left(a\left(v_{h}\right) D_{-x} u_{h}\right)\left(x_{i}, y_{j}\right)= \\
& \frac{1}{h}\left(a\left(A_{h, x} v_{h}\left(x_{i+1}, y_{j}\right)\right) D_{-x} u_{h}\left(x_{i+1}, y_{j}\right)-a\left(A_{h, x} v_{h}\left(x_{i}, y_{j}\right)\right) D_{-x} u_{h}\left(x_{i}, y_{j}\right)\right),
\end{aligned}
$$

where $D_{-x}$ denotes the backward finite difference operator with respect to the $x$-variable and $A_{h, x}$ is the following average operator

$$
A_{h, x} v_{h}\left(x_{\ell}, y_{j}\right)=\frac{1}{2}\left(v_{h}\left(x_{\ell}, y_{j}\right)+v_{h}\left(x_{\ell-1}, y_{j}\right)\right) .
$$

The finite difference operator $D_{y}^{*}\left(b\left(v_{h}\right) D_{-y} u_{h}\right)\left(x_{i}, y_{j}\right)$ is defined analogously considering the backward finite difference operator with respect to the $y$-variable, $D_{-y}$, and the average operator $A_{h, y}$. If $B$ is a diagonal matrix with entries $a$ and $b$ we use the following notation

$$
\nabla_{h}^{*} \cdot\left(B\left(v_{h}\right) \nabla_{h} u_{h}\right)=D_{x}^{*}\left(a\left(v_{h}\right) D_{-x} u_{h}\right)+D_{y}^{*}\left(b\left(v_{h}\right) D_{-y} u_{h}\right) .
$$

In $[0, T]$ we consider the following time grid

$$
\left\{t_{n}, n=0, \ldots, M_{\Delta t}, t_{0}=0, t_{M_{\Delta t}}=T, t_{n}-t_{n-1}=\Delta t, n=1, \ldots, M_{\Delta t}\right\} .
$$

By $D_{-t}$ we denote the backward finite difference operator with respect to the variable $t$. Let $p_{h}^{n}\left(x_{i}, y_{j}\right)$ stands for an approximation of $p\left(x_{i}, y_{j}, t_{n}\right)$.

To solve numerically the initial boundary value problem (1), (3), (4) we consider the IMEX method defined by

$$
\left\{\begin{array}{l}
D_{-t} C_{W, h}^{n+1}=\nabla_{h}^{*} \cdot\left(D_{W} \nabla_{h} C_{W, h}^{n+1}\right)+\nabla_{h}^{*} \cdot\left(D_{v} \nabla_{h} \sigma_{h}^{n}\right)-k C_{W, h}^{n} M_{h}^{n} \text { in } \Omega_{h}, \\
D_{-t} M_{h}^{n+1}=-k C_{W, h}^{n+1} M_{h}^{n} \text { in } \bar{\Omega}_{h}, \\
D_{-t} \sigma_{h}^{n+1}+\frac{E_{0}\left(M_{h}^{n+1}\right)^{\alpha}}{\mu_{0}\left(M_{h}^{n+1}\right)^{\beta}} \sigma_{h}^{n}=-E_{0}\left(M_{h}^{n+1}\right)^{\alpha} D_{-t} C_{W, h}^{n+1} \text { in } \bar{\Omega}_{h}, \\
D_{-t} C_{S, h}^{n+1}=-\frac{k_{d i s}}{C_{S 0} C_{A m x} C_{W o u t}} C_{S, h}^{n}\left(C_{A m x}-C_{A, h}^{n}\right) C_{W, h}^{n+1} \text { in } \bar{\Omega}_{h}, \\
D_{-t} C_{A, h}^{n+1}=\nabla_{h}^{*} \cdot\left(D\left(M_{h}^{n+1}\right) \nabla_{h} C_{A, h}^{n+1}\right) \\
\quad+\frac{k_{d i s}}{C_{S 0} C_{A m x} C_{W o u t}} C_{S, h}^{n+1}\left(C_{A m x}-C_{A, h}^{n}\right) C_{W, h}^{n+1} \text { in } \Omega_{h},
\end{array}\right.
$$

for $n=0, \ldots, M_{\Delta t}-1$,

and

$$
\left\{\begin{array}{l}
C_{W, h}^{0}=0 \text { in } \Omega_{h}, \\
\sigma_{h}^{0}=\sigma(0) \text { in } \Omega_{h}, \\
M_{h}^{0}=M(0) \text { in } \Omega_{h}, \\
C_{S, h}^{0}=C_{S}(0) \text { in } \Omega_{h}, \\
C_{A, h}^{0}=0 \text { in } \Omega_{h},
\end{array}\right.
$$

where

$$
\left\{\begin{array}{l}
J_{h}^{n+1} \cdot \eta=A_{c}\left(C_{W, h}^{n+1}-C_{W o u t}\right) \text { on } \partial \Omega_{h}, \\
C_{A, h}^{n+1}=0 \text { on } \partial \Omega_{h},
\end{array}\right.
$$

and $D_{\eta}$ is the boundary operator

$$
J_{h}^{n+1}=-D_{W} D_{\eta} C_{W, h}^{n+1}-D_{v} D_{\eta} \sigma_{h}^{n},
$$

$$
D_{\eta} v_{h}\left(x_{i}, y_{j}\right)=\left\{\begin{array}{l}
-D_{x} v_{h}\left(x_{0}, y_{j}\right), i=0, \\
D_{-x} v_{h}\left(x_{N}, y_{j}\right), i=N, \\
-D_{y} v_{h}\left(x_{i}, y_{0}\right), j=0, \\
D_{-y} v_{h}\left(x_{i}, y_{N}\right), j=N,
\end{array}\right.
$$

for $\left(x_{i}, y_{j}\right) \in \partial \Omega_{h}$. 
Table 1: Parameter values used in the numerical simulation

\begin{tabular}{llll}
\hline Parameter (unit) & Value & Parameter (unit) & Value \\
\hline$D_{A}\left(\mathrm{~mm}^{2} / \mathrm{s}\right)$ & $5.94 \times 10^{-2}$ & $E_{0}(\mathrm{~Pa})$ & $10^{-3}$ \\
$D_{v}(\mathrm{~mol} /(\mathrm{mm} . \mathrm{s} . \mathrm{Pa}))$ & $2 \times 10^{-2}$ & $\mu_{0}(\mathrm{~Pa} . \mathrm{s})$ & $10^{-1}$ \\
$D_{W}\left(\mathrm{~mm}^{2} / \mathrm{s}\right)$ & $4.61 \times 10^{-2}$ & $k_{\text {dis }}\left(\mathrm{mol} /\left(\mathrm{mm}^{3} . \mathrm{s}\right)\right)$ & $4.6 \times 10^{-2}$ \\
$k(1 / \mathrm{s})$ & $10^{-2}$ & $M_{0}(\mathrm{Da})$ & $8.3 \times 10^{-2}$ \\
$\tilde{k}\left(\mathrm{~mm}^{3} /(\mathrm{s} . \mathrm{mol})\right)$ & $10^{-2}$ & $k_{1}\left(\mathrm{~mm}^{3} / \mathrm{mol}\right)$ & 1 \\
$\sigma_{0}(\mathrm{~Pa})$ & $5 \times 10^{-2}$ & $C_{W o u t}\left(\mathrm{~mol} / \mathrm{mm}^{3}\right)$ & $5.55 \times 10^{-1}$ \\
$C_{A m x}\left(\mathrm{~mol} / \mathrm{mm}^{3}\right)$ & $2.184 \times 10^{-2}$ & $A_{c}(\mathrm{~mm} / \mathrm{s})$ & $10^{-2}$ \\
$C_{S_{0}}\left(\mathrm{~mol} / \mathrm{mm}^{3}\right)$ & $288.42 \times 10^{-2}$ & $\alpha$ & $2 \times 10^{-1}$ \\
$\beta$ & $7 \times 10^{-1}$ & $L(\mathrm{~mm})$ & 1 \\
$\Delta t$ & $10^{-4}$ & $h$ & $10^{-2}$ \\
\hline
\end{tabular}

Table 2: Errors and convergence orders for the concentration of water and dissolved drug

\begin{tabular}{lllll}
\hline$h$ & Error $\left(C_{W}\right)$ & $p_{w}$ & $\operatorname{Error}\left(C_{A}\right)$ & $p_{a}$ \\
\hline 0.01 & $3.23 \times 10^{-5}$ & 1.38 & $5.29 \times 10^{-10}$ & 1.08 \\
0.005 & $1.24 \times 10^{-5}$ & 1.35 & $2.50 \times 10^{-10}$ & 1.30 \\
0.004 & $9.17 \times 10^{-6}$ & 2.01 & $1.87 \times 10^{-10}$ & 1.61 \\
0.002 & $2.28 \times 10^{-6}$ & & $6.13 \times 10^{-11}$ & \\
\hline
\end{tabular}

\section{Qualitative behaviour of the model}

In this section we illustrate the influence of the parameters on the behaviour of the model. The values of the parameters are presented in Table 1 and some of them were obtained from [15].

We start by analyzing numerically the convergence properties of the numerical scheme. Table 2 contains the errors for $C_{W}$ and $C_{A}$ at time $T=0.001$ defined by,

$$
\operatorname{Error}(C)=\left\|C_{h}^{n}-\bar{C}_{h}^{n}\right\|_{L^{2}\left(\Omega_{h}\right)}=\left(\sum_{P \in \bar{\Omega}_{h}} q_{1}(P)\left(C_{h}^{n}(P)-\bar{C}_{h}^{n}(P)\right)^{2}\right)^{1 / 2}
$$

where $q_{1}(P)=h^{2}$ on $\Omega_{h}, q_{1}(P)=\frac{h^{2}}{2}$ on $\partial \Omega_{h}-\mathcal{C}_{h}$ and $q_{1}(P)=\frac{h^{2}}{4}$ on $\mathcal{C}_{h}$, with $\mathcal{C}_{h}=\left\{\left(x_{i}, y_{j}\right): i, j=0, N\right\}$, using a reference solution $\bar{C}_{h}^{n}$ obtained with a fine grid defined by $\Delta t=10^{-5}$ and $h=0.001$. In the same table we also present estimations for the convergence orders, using the following formula

$$
p=\frac{\ln \left(\frac{\operatorname{Error}_{h_{1}}(C)}{\operatorname{Error}_{2}(C)}\right)}{\ln \left(\frac{h_{1}}{h_{2}}\right)},
$$

where $h_{1}$ and $h_{2}$ represent two consecutive step sizes. The results suggest the convergence of the IMEX method (26)-(28) with second convergence rate in space.

Let the mass of water and drug, inside the matrix, be defined by

$$
\mathcal{M}_{i}(t)=\int_{\Omega} C_{i}(t) d x d y
$$

where $i=W, A$, for $t \in[0, T]$. A numerical approximation for $M_{i}(t)$ is computed with the trapezoidal rule.

In Figure 1 we plot the dependence on the viscoelastic diffusion coefficient $D_{v}$ of the mass of water inside the polymeric matrix. In this figure as well as in the following the time is measured in seconds. We observe that the polymer acts as a barrier to the entrance of water. In other words, the non Fickian flux 

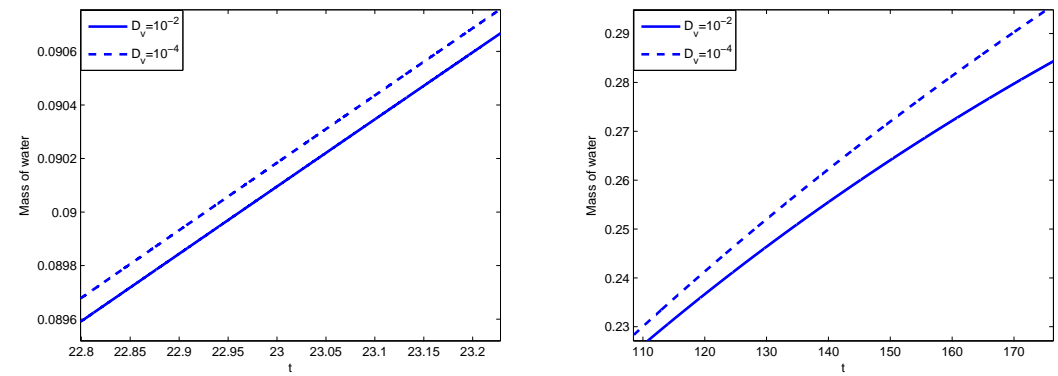

Figure 1: Influence of $D_{v}$ on the mass of the water inside the polymeric matrix.

$-D_{v} \nabla \sigma$ decreases the Fickian flux, $-D_{W} \nabla C_{W}$. According to this description an increase in $D_{v}$ leads to a increase of $M_{W}$.

The influence of the Young modulus $E$ on $M_{W}$ is presented in Figure 2 (left), near $t=2$. It is well known that the crosslink density of the polymer is proportional to the Young modulus $E$. Consequently as this constant increases the resistance of the polymer to the entrance of water also increases leading to a decreasing of the mass of water.
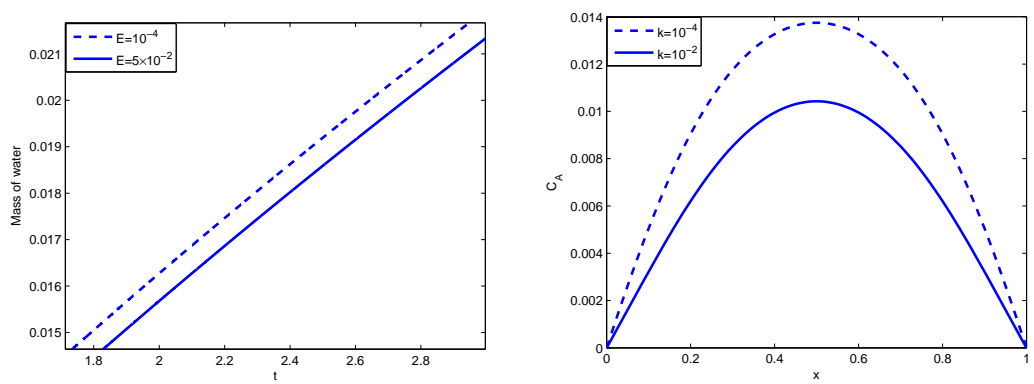

Figure 2: Mass of water for different $E$ 's (left); concentration of dissolved $\operatorname{drug} C_{A}$ for different $k$ (right).

The influence of the polymer degradation rate, $k$, is presented in Figure 2 (right). As expected, if the degradation rate increases, then the delivery rate of the dissolved drug also increases.

The behaviour of the mass of dissolved drug is presented in Figure 3, for different thickness of the polymer. We observe that the maximum value of the mass of dissolved drug in thinner polymers is lower and less time is required to achieve this maximum. The amount of dissolved drug results from a balance between the dissolution process and the release of drug. The instant when the maximum occurs, represents the time when the delivery process dominates the dissolution process.
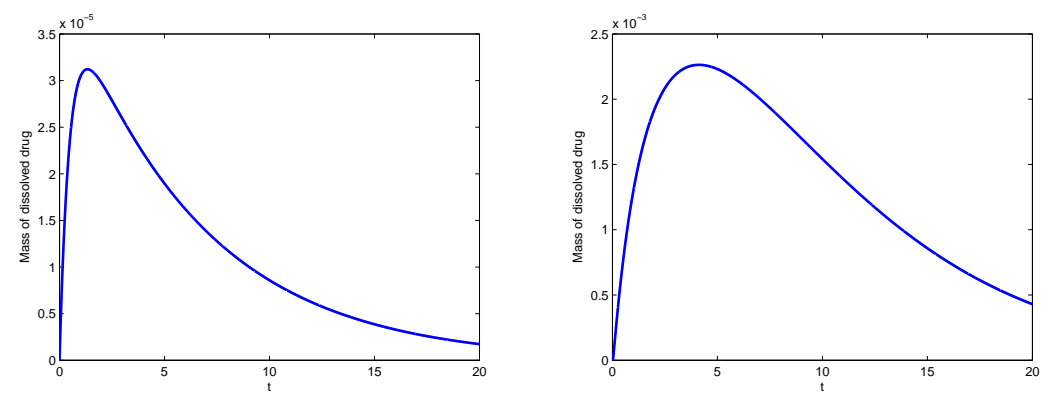

Figure 3: Mass of dissolved drug inside the polymer with $L=0.1$ (left) and $L=0.5$ (right).

In Figure 4 the mass of water inside the polymer, for different values of $L$, is plotted. In the thicker 
polymer more time is required for the mass to reach the steady state. We also observe that the value of the steady state in the polymer with $L=0.1$ is 0.0555 while in the polymer with $L=0.5$ is 0.2769 .
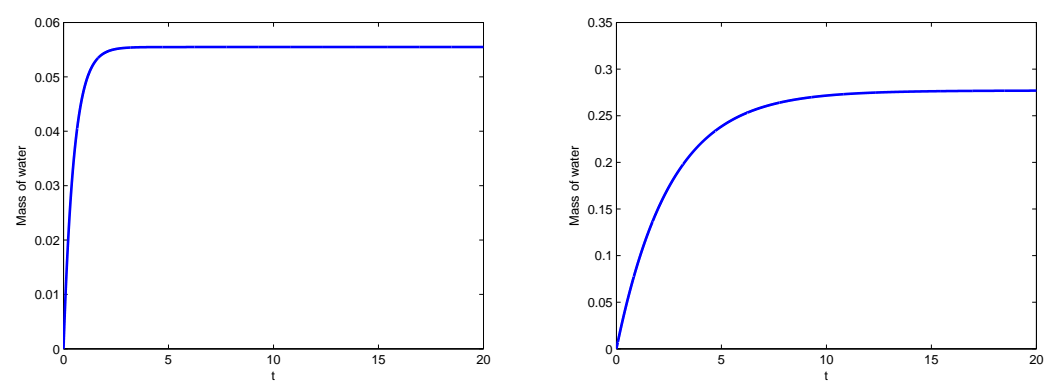

Figure 4: Mass of water inside the polymer with $L=0.1$ (left) and $L=0.5$ (right).
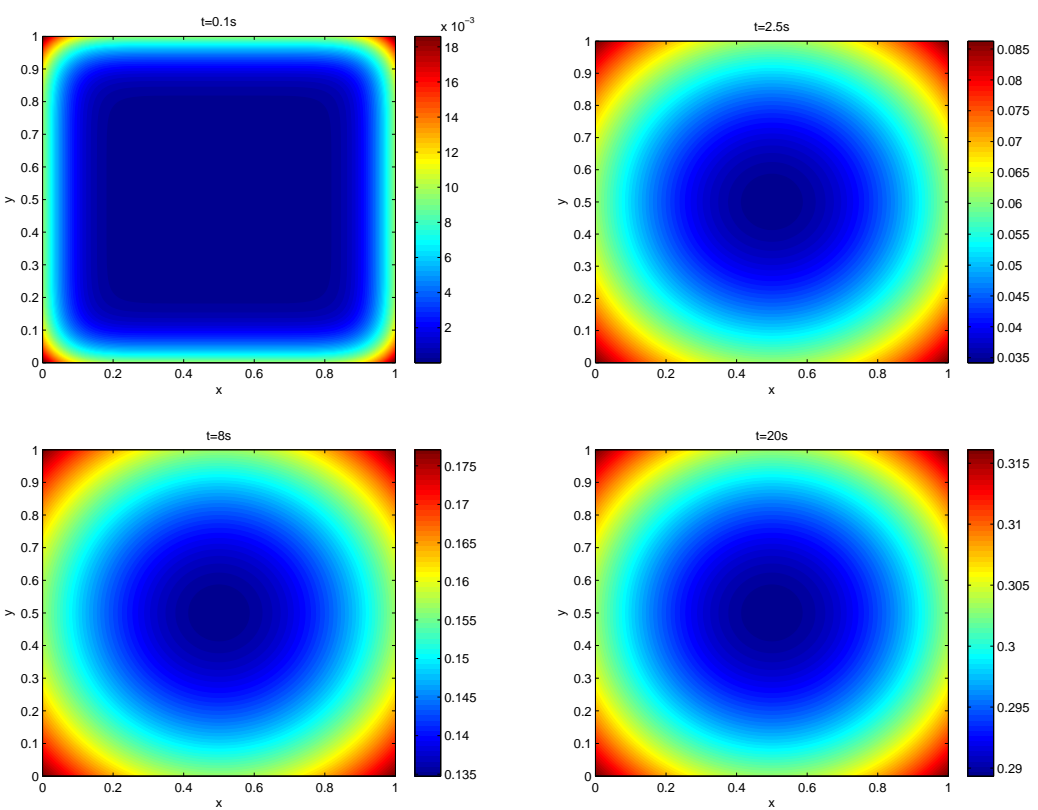

Figure 5: Concentration of water for different times.

Figure 5 illustrates the behavior of the concentration of water into the polymeric matrix at different times. We observe that the concentration increases as time increases and the behavior is homogeneous since the diffusion coefficient is constant.

The concentration of solid drug and dissolved drug, respectively, at different times are shown in Figures 6 and 7. Te regions where the concentration of water is higher, correspond to regions where the concentration of solid drug is lower. We also note that when the concentration of solid drug decreases, the concentration of dissolved drug increases.

\section{Conclusions}

In this paper we describe a process of sorption of a solvent by a biodegradable polymeric matrix, when bulk erosion occurs, and the simultaneous release of a drug. The stability analysis of the mathematical model was studied. Numerical results that highlight the whole process are presented. These results are physically sound. The influence of the crosslinking density of the polymer is shown to delay the drug release. In fact a larger Young modulus exerts a larger opposition to the solvent penetration. Bulk 

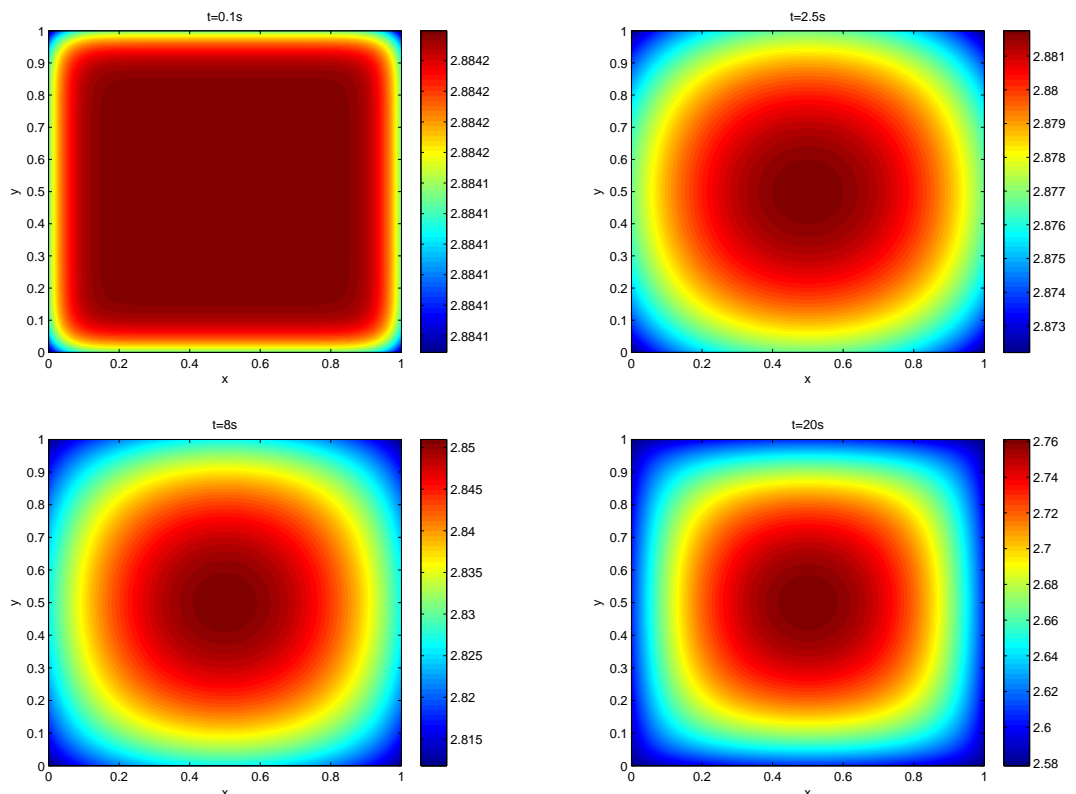

Figure 6: Concentration of solid drug for different times.
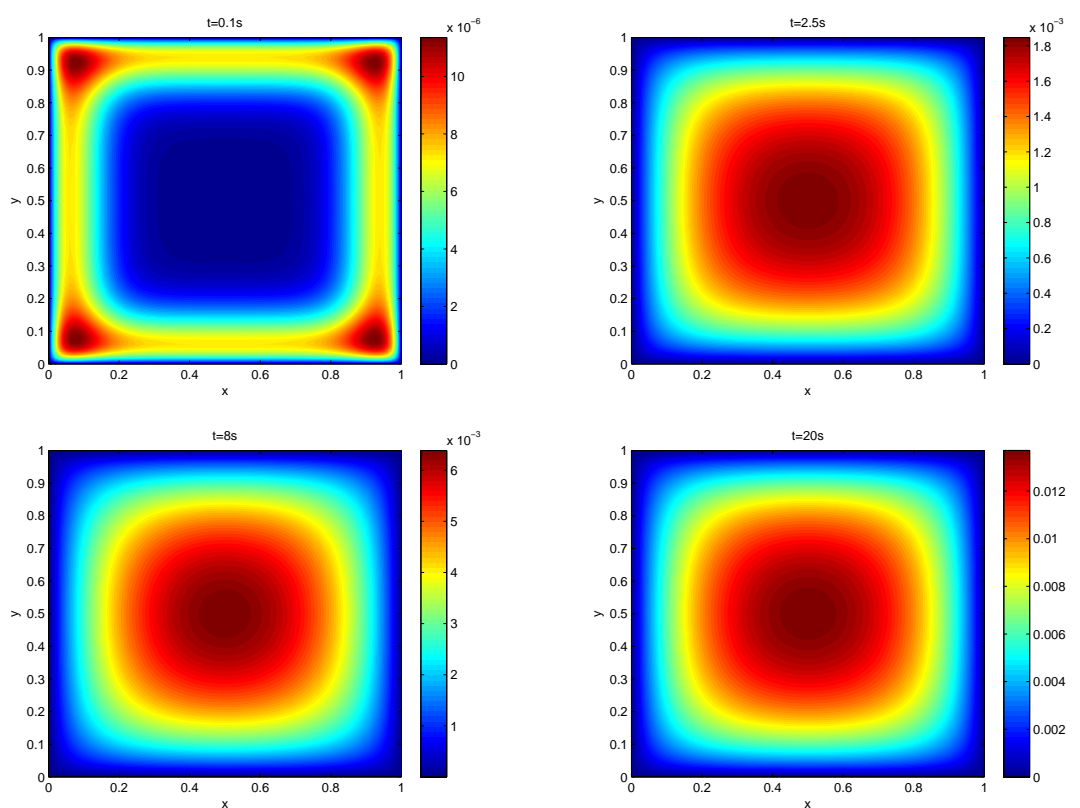

Figure 7: Concentration of dissolved drug for different times. 
erosion which is governed by the degradation rate speeds up the release of drug. The dependence on the dimensions of the matrix is also illustrated.

\section{Acknowledgements}

This work was partially supported by the Centro de Matemática da Universidade de Coimbra (CMUC), funded by the European Regional Development Fund through the program COMPETE and by the Portuguese Government through the FCT - Fundação para a Ciência e Tecnologia under the project PEst-C/MAT/UI0324/2013.

\section{References}

[1] R. Al-Itrry, K. Lamnawar, A. MaAzouz, Improvement of thermal stability, rheological and mechanical properties of PLA, PBAT and their blends by reactive extrusion with functionalized epoxy, Polym. Degrad. Stab. 97 1898-1914 (2012).

[2] M. Al-Nasassrah, F. Podczeck, J.Newton, The effect of an increase in chain length on the mechanical properties of polyethylene glycols, Eur. J. Pharm. Biopharm. 46 31-38 (1998).

[3] E. Azhdari, J.A. Ferreira, P. De Oliveira, P.M. da Silva, Dissolution and bulk erosion in viscoelastic materials: numerical study, Proceedings of the 14th International Conference on Computational and Mathematical Methods in Science and Engineering, CMMSE 2014, EditorS: J. Vigo-Aguiar, I. P. Hamilton, J. Medina, P. Schwerdtfeger, W. Sprößig, M. Demiralp, E. Venturino, V.V. Kozlov, P. Oliveira I 115-126 (2014).

[4] F. Radu, M. Bause, P.Knaber, G.Lee, W. Friess, Modeling drug release from collagen matrices Journal of Pharmaceutical Sciences 91 964-972 (2002).

[5] E. Azhdari, J.A. Ferreira, P. De Oliveira, P.M. da Silva, Drug delivery from an ocular implant into the vitreous chamber of the eye, Proceedings of the 13th International Conference on Computational and Mathematical Methods in Science and Engineering, CMMSE 2013, Editors: I. Hamilton and J. Vigo-Aguiar I 185-195 (2013).

[6] E. Azhdari, J. A. Ferreira, P. De Oliveira, P. M. Da Silva, Diffusion, viscoelasticity and erosion: analytical study and medical applications, J. Comput. Appl. Math. doi: 10.1016/j.cam.2014.01.025 (2014).

[7] S. Baruah, N. Laskar, Relation between molecular weight and viscosity for Polydispersed Pol(ndocosyl acrylate), Polym. J. 28 893-895 (1996).

[8] J.A. Ferreira, M. Grassi, E. Gudiño, P. De Oliveira, A 3D model for mechanistic control drug release, SIAM J. Appl. Math. 74 620-633 (2014).

[9] A.Gopferich, R. Langer, Modeling of polymer erosion, Macromolecules 26 4105-4112 (1993).

[10] A. Gopferich, Mechanisms of polymer degradation and erosion, Biomaterials 17 103-114 (1996).

[11] A. Izuka, H. Winter, T. Hashimoto, Molecular weight dependence of viscoelasticity of polycaprolactone critical gels, Macromolecules 25 2422-2428 (1992).

[12] S. Luo, D. T. Grubba And A. N. Netravali, The effect of molecular weight on the lamellar structure, thermal and mechanical properties of poly (hydroxybutyrate-co-hydroxyvalerates), Polymer 43 4159-4166 (2002).

[13] Y. Wang, X. Han, J. Pan, C. Sinka, An entropy spring model for the Young's modulus change of biodegradable polymers during biodegradation, J. Mech. Behav. Biomed. 3 14-21 (2010). 
[14] S. Luo, D. Grubba, And A. Netravali, The effect of molecular weight on the lamellar structure, thermal and mechanical properties of poly (hydroxybutyrate-co-hydroxyvalerates), Polymer 43 41594166 (2002).

[15] S. N. Rothstein, W. J. Federspiel, S. R. Little, A unified mathematical model for the prediction of controlled release from surface and bulk eroding polymer matrices, Biomaterials 30 1657-1664 (2009).

[16] T. Rushing, R. Hester, Intrinsic viscosity dependence on polymer molecular weight and fluid temperature, J. Appl. Polym. Sci. 89 2831-2835 (2003).

[17] J. Siepmann, A. GöPferich, Mathematical modeling of bioerodible polymeric drug delivery systems, Adv. Drug. Deliver Rev. 48 229-247 (2001).

[18] J. Torres, C. StafFord, B. Vogt, Impact of molecular mass on the elastic modulus of polystyrene thin films, Polymers 51 4211-4217 (2010). 